\title{
The Robust Set problem: parameterized complexity and approximation
}

\author{
Cristina Bazgan ${ }^{1,2}$ and Morgan Chopin ${ }^{1}$ \\ 1 Université Paris-Dauphine, LAMSADE, France \\ \{bazgan, chopin\}@lamsade. dauphine.fr \\ 2 Institut Universitaire de France
}

\begin{abstract}
In this paper, we introduce the $k$-Robust SET problem: given a graph $G=(V, E)$, a threshold function $t: V \rightarrow N$ and an integer $k$, find a subset of vertices $V^{\prime} \subseteq V$ of size at least $k$ such that every vertex $v$ in $G$ has less than $t(v)$ neighbors in $V^{\prime}$. This problem occurs in the context of the spread of undesirable agents through a network (virus, ideas, fire, ...). Informally speaking, the problem asks to find the largest subset of vertices with the property that if anything bad happens in it then this will have no consequences on the remaining graph. The threshold $t(v)$ of a vertex $v$ represents its reliability regarding its neighborhood; that is, how many neighbors can be infected before $v$ gets himself infected.

We study in this paper the parameterized complexity of $k$-RoBUst SET and the approximation of the associated maximization problem. When the parameter is $k$, we show that this problem is $\mathrm{W}[2]$-complete in general and W[1]-complete if all thresholds are constant bounded. Moreover, we prove that, if $P \neq N P$, the maximization version is not $n^{1-\epsilon}$ - approximable for any $\epsilon>0$ even when all thresholds are at most two. When each threshold is equal to the degree of the vertex, we show that $k$ RoBust SET is fixed-parameter tractable for parameter $k$ and the maximization version is APX-complete. We give a polynomial-time algorithm for graphs of bounded treewidth and a PTAS for planar graphs. Finally, we show that the parametric dual problem $(n-k)$-RoBUST SET is fixedparameter tractable for a large family of threshold functions.
\end{abstract}

\section{Introduction}

The subject of optimization problems that involve a diffusion process through a network is a large and well-studied topic $[15,8,1,12,3]$. Such problems share a common idea of selecting an initial subset of vertices to activate in a graph such that, according to a propagation rule, all vertices are activated once the propagation process stops. One such representative problem is the TARGET SET SELECTION problem first introduced in [8]: given a graph $G=(V, E)$ and a threshold function $t: V \rightarrow N$, the problem asks to find the minimum number of vertices to activate such that all vertices are activated at the end of the propagation process. A vertex $v$ is activated if and only if the number of its activated neighbors is above the threshold $t(v)$. This problem has been proved 
NP-complete even when all thresholds are at most two [8]. Moreover, the problem was surprisingly shown to be hard to approximate within a ratio $O\left(2^{\log ^{1-\epsilon} n}\right)$ for any $\epsilon>0$ even when all thresholds are at most two [8]. The same inapproximability result holds with majority thresholds i.e, for each $v \in V, t(v)=\left\lceil\frac{d(v)}{2}\right\rceil$ [8]. From a parameterized perspective, Target Set Selection is W[2]-hard with respect to the solution size for majority thresholds and for thresholds at most two [17]. Furthermore, the problem is proved to be in XP and W[1]-hard with respect to the treewidth [3]. These negative results emphasis the strong intractability nature of the problem.

With regards to the motivation for this study, it is an interesting question to ask the complexity of the converse problem: find the largest subset of vertices such that if a set of vertices gets infected in it then there will be no consequence for all the other vertices. In this context, there is no propagation process involved like in the previous problem; here we want to prevent such phenomenon. This idea of "controlling" the diffusion of dangerous ideas or epidemics is also wellstudied $[16,13,7]$. More formally, we introduce the $k$-Robust SET problem: given a graph $G=(V, E)$, a threshold function $t: V \rightarrow N$ and an integer $k$, find a subset of vertices $V^{\prime} \subseteq V$ of size at least $k$ such that every vertex $v$ in $G$ has less than $t(v)$ neighbors in $V^{\prime}$. The set $V^{\prime}$ is said to be robust. Indeed, if one infects any subset $S \subseteq V^{\prime}$ then there will be no propogation at all since every vertex has a number of infected neighbors below its threshold. Finally, it is worth pointing out that our problem can also be related to a recent paper about the $(\sigma, \rho)$-Dominating SET problem [14].

In this paper, we study the parameterized complexity of $k$-RoBUST SET and the approximation of the associated maximization problem Max RoBUst SeT. The paper is organized as follows. In Section 2 we give the definitions, terminology and preliminaries. In Section 3 we establish parameterized intractability results for $k$-RoBust SET with various threshold functions. We show that the parametric dual problem $(n-k)$-RoBUST SET is fixed-parameter tractable for a large family of threshold functions. In Section 4 we give a polynomial-time algorithm to solve $k$-RoBUst SeT for graphs of bounded treewidth. In Section 5 we establish that Max Robust Set is not $n^{1-\epsilon}$-approximable for any $\epsilon>0$ even when all thresholds are at most two. If each threshold is equal to the degree of the vertex, we show that Max RoBust SET is APX-complete. Conclusion and open problems are given in Section 6. Due to the space limit, some proofs are omitted.

\section{Preliminaries}

In this section, we give the notation used throughout this paper as well as the statement of the problems. We will conclude this section by providing the basic backgrounds on parameterized complexity and approximation.

Graph terminology. Let $G=(V, E)$ be an undirected graph. The open neighborhood of a node $v \in V$, denoted by $N(v)$, is the set of all neighboors of $v$. The 
closed neighborhood of a node $v$, denoted $N[v]$, is the set $N(v) \cup\{v\}$. The degree of a node $v$ is denoted by $d_{G}(v)$ or simply $d(v)$ if the graph is clear from context. Let $X \subseteq V$, we denote by $G[X]$ the subgraph of $G$ induced by $X$.

Problem definitions. Let $G=(V, E)$ be an undirected graph, a threshold function $t: V \rightarrow N$. A subset $V^{\prime} \subseteq V$ is called robust if $\forall v \in V, d_{V^{\prime}}(v)<t(v)$. We call a vertex bounded if $t(v)=O(1)$; otherwise, it is said unbounded. We define in the following the problems we study in this paper.

$k$-RobUST SET

Input: A graph $G=(V, E)$, a threshold function $t: V \rightarrow N$ where $1 \leq t(v) \leq d(v)$ for every $v \in V$, and an integer $k$

Parameter: $k$

Question: Is there a robust set $V^{\prime} \subseteq V$ of size at least $k$ ?

We considered also the parametric dual problem $(n-k)$-RoBust SET which asks for the existence of a robust set of size at least $n-k$.

The optimization version of $k$-RoBUST SET is defined as follows.

Max Robust SeT

Input: A graph $G=(V, E)$ and a threshold function $t: V \rightarrow N$ where $1 \leq t(v) \leq d(v)$ for every $v \in V$

Output: A robust set $V^{\prime} \subseteq V$ such that $\left|V^{\prime}\right|$ is maximized.

If the threshold function is defined by $t(v)=d(v), \forall v \in V$ then we add the suffix With UnAnimity to the problem name. The majority threshold is $t(v)=\left\lceil\frac{d(v)}{2}\right\rceil, \forall v \in V$.

Parameterized complexity. Here we only give the basics notions on parameterized complexity, for more background the reader is referred to $[11,18]$. A decision problem parameterized by $k$ is said to be fixed-parameter tractable if there exists an algorithm that solves every instance $(I, k)$ in fpt-time i.e., in $f(k) \cdot|I|^{O(1)}$-time for some function $f$ depending solely on $k$.

The basic class of parameterized intractability is $\mathrm{W}[1]$ and there is a good reason to believe that $\mathrm{W}[1]$-hard problems are unlikely to be fixed-parameter tractable. In fact, there is a all hierarchies of classes $\mathrm{W}[\mathrm{i}]$ with the following inclusions $\mathrm{FPT} \subseteq \mathrm{W}[1] \subseteq \mathrm{W}[2] \ldots$. A problem in $\mathrm{W}[i]$ is considered harder than those in $\mathrm{W}[i-1]$ where $i>1$. These classes are defined via the satisfiability problem of boolean circuits. More specifically, a parameterized problem belongs to $\mathrm{W}[\mathrm{i}]$ if every instance $(I, k)$ can be transformed in fpt-time to a boolean circuit $C$ of constant depth and weft at most $i$, such that $(I, k)$ is a yes instance if and only if there is a satisfying truth assignment for $C$ of weight exactly $k$. The weft of a circuit is the maximum number of large gates i.e., gates with a number of inputs not bounded by any constant, on a path from an input to the output. The depth is the maximum number of all gates on a path from an input to the output.

In this paper, the kernel size is expressed in terms of the number of vertices. 
Approximation. Given an optimization problem $A$ and an instance $I$ of this problem, we denote by $|I|$ the size of $I$, by $\operatorname{opt}_{A}(I)$ the optimum value of $I$ and by $\operatorname{val}(I, S)$ the value of a feasible solution $S$ of $I$. In this paper, we will make use of the following approximation preserving reduction.

Definition 1 ( $L$-reduction [19]). Let $A$ and $B$ be two optimization problems. Then $A$ is said to be L-reducible to $B$ if there are two constants $\alpha, \beta>0$ and two polynomial time computable functions $f, g$ such that

1. $f$ maps an instance $I$ of $A$ into an instance $I^{\prime}$ of $B$ such that $\operatorname{opt}_{B}\left(I^{\prime}\right) \leq$ $\alpha \cdot \mathrm{opt}_{A}(I)$,

2. $g$ maps solutions each solution $S^{\prime}$ of $I^{\prime}$ into a solution $S$ of $I$ such that $\left|\operatorname{val}(I, S)-\operatorname{opt}_{A}(I)\right| \leq \beta \cdot\left|\operatorname{val}\left(I^{\prime}, S^{\prime}\right)-o p t_{B}\left(I^{\prime}\right)\right|$.

For us the important property of this reduction is that if $A$ is APX-hard then $B$ is also APX-hard.

\section{Parameterized complexity}

In this section, we consider the parameterized complexity of $k$-RoBUST SET. In some reductions we make use of the following gadget: a forbidden edge denotes an edge $u v$ where both vertices have threshold one. Attaching a forbidden edge to a vertex $w$ means to create a forbidden edge $u v$ and make $w$ adjacent to $u$. Notice that none of the three vertices $u, v$ or $w$ can be part of a robust set.

First, we show that $k$-Robust Set belongs to W[2] using the Turing way, that is, we reduce $k$-Robust Set to the Short Multi-Tape NondeterminISTIC TURING MaChine problem that is proved to belong to $\mathrm{W}[2]$ in $[6]$ and defined as follows: given a multi-tape nondeterministic Turing machine $M$, a word $x$ on the input alphabet of $M$, and an integer $k$, determine if there is a computation of $M$ on input $x$ that reaches a final accepting state in at most $k$ steps. The parameter is $k$.

Theorem 1. $k$-Robust SET is in W[2].

Proof. We construct an fpt-reduction from $k$-Robust Set to Short Multitape Nondeterministic Turing Machine as follows. Let $(G, t, k)$ be an instance of $k$-Robust SET with $G=(V, E)$ and $V=\left\{v_{1}, \ldots, v_{n}\right\}$. We construct the following Turing machine $M$ from $(G, t, k)$. We create $n+1$ tapes denoted by $T_{0}, T_{v_{1}} \ldots, T_{v_{n}}$. The tapes alphabet is $V \cup\{\times, 1, \ldots, n\}$ plus the blank symbol 0 . Initially, every tape is filled with 0 . The transition function is defined hereafter. The machine $M$ starts by writing symbol $\times$ on tape $T_{0}$ and move $T_{0}$ 's head one step to the right. During the first phase, $M$ non-deterministically chooses $k$ vertices and write them on tape $T_{0}$, that is, if $M$ picks up a vertex $v \in V$ then it writes symbol $v$ on $T_{0}$ and move $T_{0}$ 's head one step to the right. The previous procedure is done in $k+1$ steps. During the second phase, $M$ verifies that the selected set is a robust set as follows. First, the machine move $T_{0}$ 's head one step to the left. Assume that $T_{0}$ 's head reads symbol $v$ and, for every $u \in N(v), T_{u}$ 's 
head reads symbol $s_{u}$. If $s_{u}=t(u)-1$ then $M$ goes in rejecting state. Otherwise, $M$ writes symbol $s_{u}+1$ on tape $T_{u}$ and moves the $T_{0}$ 's head one step to the left. We repeat the previous procedure until $T_{0}$ 's head reads symbol $\times$. Clearly, this checking phase is performed in at most $k+1$ steps. Finally, the input word $x$ is empty and $k^{\prime}=2 k+2$. It is not hard to see that $(G, t, k)$ is a Yes-instance if and only if $M$ accepts in at most $k^{\prime}$ steps.

Now in order to prove the W[2]-hardness of $k$-Robust SET, we construct a simple fpt-reduction from the problem Red/Blue Dominating Set proved $\mathrm{W}[2]$-hard in [11] and defined as follows: given a bipartite graph $G=(R \cup B, E)$ and a positive integer $k$, determine if there exists a set $R^{\prime} \subseteq R$ of cardinality $k$ such that every vertex in $B$ has at least one neighbor in $R^{\prime}$. The parameter is $k$.

Theorem 2. $k$-RoBUST SET is W[2]-complete even for bipartite graphs.

Proof. Membership follows from Theorem 1. Now, let us show the W[2]-hardness. Given $(G, k)$ an instance of Red/Blue Dominating Set, we construct an instance $\left(G^{\prime}=\left(V^{\prime}, E^{\prime}\right), t, k\right)$ of $k$-Robust SET as follows. We consider the complement $\bar{G}$ of the graph $G$, that is two vertices $u \in R$ and $v \in B$ are adjacent in $\bar{G}$ if and only if they are not adjacent in $G$. Moreover, the sets $R$ and $B$ remain independent sets. Graph $G^{\prime}$ is obtained from this last graph by attaching $\max \left\{k-d_{\bar{G}}(v), 1\right\}$ forbidden edges to each vertex $v \in B$. Finally, set $t(v)=k$ for every vertex $v \in B$ and $t(v)=1$ for every vertex $v \in R$. Adding several forbidden edges to the vertices of $B$ make sure that the threshold of these vertices is less than or equal to their degree as required.

Assume that $(G, k)$ has a solution $R^{\prime} \subseteq R$ of size $k$. One can see that $R^{\prime}$ is also a solution for $\left(G^{\prime}, t, k\right)$ since every vertex in $B$ is not adjacent to at least one vertex in $R^{\prime}$. Conversely, suppose that there is a robust set $S \subseteq V^{\prime}$ of size $k$ in $G^{\prime}$. Since $S$ is robust, $S$ cannot contain any vertex from $B$ because of the forbidden edges, and thus $S$ is entirely contained in $R$. Moreover, every vertex $v$ in $B$ is adjacent in $G^{\prime}$ to at most $t(v)-1=k-1$ vertices in $S$. Hence, every vertex in $B$ is adjacent in $G$ to at least one vertex in $S$. Therefore, $S$ is a solution of size $k$ for $(G, k)$.

In the next two theorems, we show that $k$-RoBUST SET goes one level down in the W-hierarchy when all thresholds are bounded by a constant.

Theorem 3. $k$-RoBUST SET is in W[1] if all thresholds are constant bounded.

Proof. Let $(G, t, k)$ be an instance of $k$-Robust SeT where $t(v) \leq c, \forall v \in V$ for some constant $c>0$. We construct in $O\left(n^{c}\right)$-time, where $n$ is the number of vertices of $G$, a boolean circuit $C$ of depth 3 and weft 1 as follows. We identify the inputs of the circuit with the vertices of $G$. Connect a $\neg$-gate to every input. For all $v \in V$ and all subsets $S^{\prime} \subseteq N(v)$ of size $t(v)$, add a $\vee$-gate connected to $\neg$-gate of inputs in $S^{\prime}$. Finally, add a large $\wedge$-gate connected to every $\vee$-gate. It is not hard to see that $G$ admits a robust set of size $k$ if and only if there is a weight- $k$ assignment that satisfies $C$. 
We establish the W[1]-hardness of $k$-RoBust SET by an fpt-reduction from the problem RED/Blue NonBlocker [10] defined as follows: given a bipartite graph $G=(R \cup B, E)$ and a positive integer $k$, determine if there is a set $R^{\prime} \subseteq R$ of cardinality $k$ such that every vertex in $B$ has at least one neighbor that does not belong to $R^{\prime}$. The parameter is $k$. This problem remains W[1]-hard even when every vertex in $B$ has degree two and every vertex of $R$ has degree at least two $[10]$.

Theorem 4. $k$-Robust SET is W[1]-complete even

1. For bipartite graphs and constant majority threshold.

2. For split graphs and constant threshold $t(v)=2, \forall v \in V$.

Proof. Membership follows from Theorem 3. We now prove the W[1]-hardness.

(1): Let $(G, k)$ be an instance of RED/Blue NonBlocker where vertices in $B$ have degree two, we construct the graph $G^{\prime}=\left(V^{\prime}, E^{\prime}\right)$ from $G$ as follows. For each vertex $v \in B$, attach a forbidden edge to $v$. Set $t(v)=\left\lceil d_{G^{\prime}}(v) / 2\right\rceil$ for all $v \in V^{\prime}$.

Assume that $(G, k)$ has a solution $R^{\prime} \subseteq R$ of size $k$. It is not hard to see that $R^{\prime}$ is also a solution for $\left(G^{\prime}, t, k\right)$. Conversely, suppose that there is a robust set $S \subseteq V^{\prime}$ of size $k$ in $G^{\prime}$. Because of the forbidden edges, the set $S$ is entirely contained in $R$. Since $R$ is a robust set, every vertex in $B$ is adjacent to at least one vertex in $R \backslash S$. Therefore, $S$ is a solution of size $k$ for $(G, k)$.

(2): Let $(G, k)$ be an instance of Red/Blue NonBlocker where vertices in $B$ have degree two and every vertex of $R$ has degree at least 2 , we construct the graph $G^{\prime}=\left(V^{\prime}, E^{\prime}\right)$ from $G$ as follows. Add edges to make $B$ a clique. Set $t(v)=2$ for all $v \in V$ and $k^{\prime}=k$. Without loss of generality we may assume that $k \geq 2$.

Assume that $(G, k)$ has a solution $R^{\prime} \subseteq R$ of size $k$. One can easily verify that $R^{\prime}$ is a robust set of size $k^{\prime}$ for $\left(G^{\prime}, k^{\prime}\right)$. Conversely, suppose that there is a robust set $S \subseteq V^{\prime}$ of size $k^{\prime}$ in $G^{\prime}$. Notice that $S \cap B=\emptyset$ since otherwise we would not have been able to take more than one vertex in $G$. Indeed, if there are two vertices $u, v \in S$ with $v \in B$ then there is always a vertex $w \in B-\{u, v\}$ adjacent to both $v$ and $u$. Thus, $S$ is entirely contained in $R$. From now, it is not hard to see that $R$ is also a solution for $(G, k)$.

It is interesting to note that the ratio between the number of unbounded vertices and the number of bounded vertices of the graph in the proof of Theorem 2 can be made arbitrarily small (add many forbidden edges). This implies a sharp dichotomy between the W[2]- and W[1]-completeness of $k$-RoBUST SET regarding the thresholds.

Unanimity threshold. We consider now the $k$-RoBUST SET WITH UNANIMITY problem. First, we start with the following easy observation. In the case of unanimous threshold, any robust set is the complement of a total dominating set. Recall that a total dominating set $S$ is a set of vertices such that every vertex has at least one neighbor in $S$. Moreover, we have the following theorem. 
Theorem 5. [9] If $G$ is a connected graph of order at least 3 then there is a total dominating set of size at most $2 n / 3$.

This implies that Max RoBUst SeT With UnAnimity always has a solution of size at least $n / 3$ when $n \geq 3$. The consequence of this result is that we directly get a linear kernel of size $3 k$. Indeed, let $(G, t, k)$ be an instance of $k$-RoBust Set With Unanimity, if $k \leq n / 3$ then the answer is Yes. If $k>n / 3$ then the instance $(G, t, k)$ is a kernel of size at most $3 k$. However, the parameter $k$ is "large" in this last case. This suggests to look for other parameterizations.

Parametric dual. Now, we show that $(n-k)$-ROBUST SET is FPT with respect to parameter $k$ for a large family of threshold functions.

Reduction rule 1 Let $(G, t, k)$ be an instance of $(n-k)$-Robust SET. If there is a vertex $v$ such that $d(v) \geq k+t(v)-1$ then remove $v$ and decrease by one the threshold of every vertex in $N(v)$ to get a new equivalent instance $\left(G^{\prime}, t^{\prime}, k\right)$.

Lemma 1. Reduction rule 1 is sound.

Proof. Let $S \subseteq V$ be a robust set of size at least $n-k$. If there is a vertex $v$ with $d(v) \geq k+t(v)-1$ then $v$ must be in $S$ since otherwise $v$ has at most $k-1$ neighbors in $V \backslash S$ and then at least $t(v)$ neighbors in $S$.

Theorem 6. $(n-k)$-RoBUST SET admits a kernel of size $O\left(k^{2}\right)$ if for all $v \in V$ $t(v)=\left\lceil\alpha_{v} d(v)^{\beta_{v}}+\gamma_{v}\right\rceil$ for any constants $\alpha_{v}, \beta_{v} \in[0,1], \alpha_{v} \beta_{v} \neq 1$, and $\gamma_{v} \in Q$.

Proof. Let $(G, t, k)$ be an instance of $(n-k)$-RoBust SET. Exhaustively apply reduction rule 1 to get $\left(G^{\prime}, t^{\prime}, k\right)$. Assume that there exists a solution $S \subseteq V$ of size at least $n-k$. Because of reduction rule 1 , we have that

$$
d(v)<k+t(v)-1=k+\left\lceil\alpha_{v} d(v)^{\beta_{v}}+\gamma_{v}\right\rceil-1 \leq k+\alpha_{v} d(v)^{\beta_{v}}+\gamma_{v}
$$

We claim that $d(v) \leq \theta_{v}(k)$ for all $v \in V^{\prime}$ where $\theta_{v}(k)=\frac{k+\gamma_{v}}{1-\alpha_{v}}+\left(1 / \beta_{v}\right)^{\frac{1}{1-\beta_{v}}}$ if $\alpha_{v} \neq 1, \frac{k+\gamma_{v}}{1-\beta_{v}}+\left(1 / \beta_{v}\right)^{\frac{1}{1-\beta_{v}}}$ otherwise. Consider the following cases.

Case 1. If $\beta_{v}=0$ then obviously $d(v) \leq \theta_{v}(k)$

Case 2. If $\beta_{v}=1$ then $d(v)<\frac{k+\gamma_{v}}{1-\alpha_{v}}<\theta_{v}(k)$ (since $\alpha_{v}<1$ )

Case 3. Suppose now that $\beta_{v} \in(0,1)$. First, it is not hard to show that the following holds: $n^{\beta_{v}} \leq \beta_{v} n$ if and only if $n \geq\left(1 / \beta_{v}\right)^{\frac{1}{1-\beta_{v}}}$ for any $n \geq 1$ and $\beta_{v} \in(0,1)$. Hence, If $d(v) \geq\left(1 / \beta_{v}\right)^{\frac{1}{1-\beta_{v}}}$ then we have $d(v) \leq k+\alpha_{v} \beta_{v} d(v)+\gamma_{v}$ and thus $d(v) \leq \frac{k+\gamma_{v}}{1-\alpha_{v} \beta_{v}} \leq \theta_{v}(k)$. Otherwise $d(v)<\left(1 / \beta_{v}\right)^{\frac{1}{1-\beta_{v}}} \leq \theta_{v}(k)$.

Since every vertex from $S$ has at least one neighbor in $V^{\prime}-S$ then $|S|$ has at most $\left|V^{\prime}-S\right| d^{\max } \leq k \theta^{\max }(k)$ vertices where $\theta^{\max }(k)=\max _{v \in V^{\prime}} \theta_{v}(k)$ and $d^{\max }$ is the maximum degree of vertices in $V^{\prime}-S$.

The kernelization procedure is then defined as follows. From an instance $(G, t, k)$ of $(n-k)$-RoBUst SET, exhaustively apply reduction rule 1 to get an instance $\left(G^{\prime}, t^{\prime}, k\right)$. If $\left|V^{\prime}\right|>k \theta^{\max }(k)+k$ then return a trivial No-instance. Otherwise, return the instance $\left(G^{\prime}, t^{\prime}, k\right)$.

Notice that if $\alpha_{v}=\beta_{v}=1$ and $\gamma_{v}=0, \forall v \in V$ then the $(n-k)$-Robust Set problem is exactly the Total Dominating Set problem which is known to be W[2]-hard [14]. 


\section{Algorithm for tree-like graphs}

In this section we establish an $O\left(T^{2 \omega} n\right)$ algorithm for MAx RoBust SET and a $O\left((k+1)^{2 \omega} n\right)$ for $k$-RoBust SET where $T$ is the maximum threshold and $\omega$ the treewidth of the input graph.

Using a nice tree decomposition together with a dynamic programming algorithm we can prove the following.

Theorem 7. Max RoBust SET is solvable in time $O\left(T^{2 \omega} n\right)$ where $T$ is the maximum threshold and $\omega$ is the treewidth of the input graph.

Now we show that $k$-RoBust SET is solvable in $O\left((k+1)^{2 \omega} n\right)$ time. For that purpose, we introduce the following reduction rule.

Reduction rule 2 Let $(G, t, k)$ be an instance of $k$-ROBUST SET. If there is a vertex $v$ such that $t(v)>k+1$ then set the threshold $t(v)$ to $k+1$ to get a new equivalent instance $\left(G, t^{\prime}, k\right)$.

Lemma 2. Reduction rule 2 is sound.

Proof. Let $(G=(V, E), k, t)$ be an instance of $k$-Robust SET. Exhaustively apply Reduction Rule 2 on $(G, t, k)$ to get a new instance $\left(G, t, k^{\prime}\right)$. It is not hard to see that if $S \subseteq V$ is a robust set of size at least $k$ for $(G, t, k)$, then any subset of size $k$ of $S$ is a robust set for $\left(G, t, k^{\prime}\right)$. The converse is clear.

We are now ready to prove the following.

Theorem 8. $k$-RoBust SET is solvable in time $O\left((k+1)^{2 \omega} n\right)$ where $\omega$ is the treewidth of the input graph.

Proof. Let $(G, t, k)$ be an instance of $k$-Robust Set. Exhaustively apply Reduction Rule 2 on $(G, t, k)$ to get a new instance $\left(G, t, k^{\prime}\right)$. Apply the algorithm from Theorem 7 on $\left(G, t^{\prime}\right)$ to get the optimal solution of value opt. If opt $\geq k$ return YES; otherwise return No. Since every threshold is at most $k+1$, the running time is $O\left((k+1)^{2 \omega} n\right)$.

Notice that if all thresholds are constant bounded then $k$-RoBUST SET is in FPT with respect to parameter treewidth.

\section{$5 \quad$ Approximability}

In this section, we show that MAx RoBUST SET is inapproximable even for small constant thresholds. In order to prove this result, we consider the MAX CLIQUE problem: given a graph $G=(V, E)$, find a clique $C \subseteq V$ of maximum size.

Theorem 9. If $N P \neq Z P P$, MAX RoBUST SET is not approximable within $n^{\epsilon}$ for any $\epsilon>0$ even for thresholds at most two. 
We now prove the APX-completeness of Max Robust Set With UnAnimITY.

Lemma 3. Max Robust Set With Unanimity is 3-approximable in polynomial time.

Proof. The algorithm consists of the following two steps:

1. Compute a spanning tree $T$ of $G$.

2. Compute an optimal solution $S$ of $T$.

Using Theorem 7, the algorithm runs in polynomial-time. Clearly, any feasible solution for $T$ is also a solution for $G$. Moreover, using Theorem 5 , we have $|S| \geq n / 3 \geq \operatorname{opt}(G) / 3$.

Theorem 10. Max Robust Set With Unanimity is APX-complete.

Proof. Membership follows from Lemma 3. In order to prove the APX-hardness we provide an L-reduction (see Definition 1) from MAX E2SAT-3 proved APXhard in [4] and defined as follows: given a CNF formula $\phi$ with $n$ variables and $m$ clauses, in which every clause contains exactly two literals and every variable appears in exactly three clauses, determine an assignment to the variables satisfying a maximum number of clauses. Notice that $m=3 n / 2$.

Given a formula $\phi$ of MAx E2SAT-3, we construct an instance $I=(G=$ $(V, E), t, k)$ of Max Robust Set With Unanimity as follows (see Figure 1$)$. For every variable $x_{i}$, we construct the complete bipartite graph $K_{3,3}\left(x_{i}\right)=$ $\left(V^{-}\left(x_{i}\right), V^{+}\left(x_{i}\right)\right)$ in which every edge $u v$ is replaced by an edge-vertex $e_{u v}$ and two edges $u e_{u v}$ and $e_{u v} v$. We denote by $E\left(x_{i}\right)$ this set of edge-vertices. The vertices in $V^{+}\left(x_{i}\right)$ (resp. $V^{-}\left(x_{i}\right)$ ) represents the positive (resp. negative) literals of $x_{i}$. We denote by $A$ the set of all vertices added so far. For every clause $c_{j}$ in $\phi$ add two adjacent clause-vertices $\bar{c}_{j}$ and $\bar{c}_{j}^{\prime}$. For every variable $x_{i}$, if $x_{i}$ appears positively (resp. negatively) in a clause $c_{j}$ then add an edge between $\bar{c}_{j}^{\prime}$ and a vertex of $V^{-}\left(x_{i}\right)$ (resp. $V^{+}\left(x_{i}\right)$ ). Thus, vertex $\bar{c}_{j}$ represents the complement of the clause $c_{j}$ in $\phi$. Finally, add two adjacent vertices $c$ and $c^{\prime}$. For every vertex $v \in V^{-}\left(x_{i}\right) \cup V^{+}\left(x_{i}\right)$, if $v$ is not adjacent to a clause-vertex then add the edge $v c^{\prime}$.

The optimal value in $I$ is bounded by the number of vertices of $G$ and thus, $\operatorname{opt}(I) \leq 15 n+2 m+2 \leq 16 \operatorname{opt}(\phi)+2 \leq 18 \operatorname{opt}(\phi)$ since $\operatorname{opt}(\phi) \geq 3 / 4 m$ and $\operatorname{opt}(\phi) \geq 1$.

Moreover, let $x^{*} \subseteq V$ be an optimal assignment for $\phi$ and let

$S=\cup_{x_{i}^{*}=1} V^{+}\left(x_{i}\right) \cup \cup_{x_{i}^{*}=0} V^{-}\left(x_{i}\right) \cup \cup_{i=1}^{n} E\left(x_{i}\right) \cup\left\{\bar{c}_{j}: c_{j}\right.$ is satisfied by $\left.x^{*}\right\} \cup\{c\}$.

We can easily verify that $S$ is a robust set and $\mid S \cap\left(V^{-}\left(x_{i}\right) \cup V^{+}\left(x_{i}\right) \cup E\left(x_{i}\right) \mid=\right.$ 12 and thus $|S \cap A|=8 m$ and then $\operatorname{opt}(I) \geq|S|=8 m+\operatorname{opt}(\phi)+1$.

Let $S$ be a robust set for $I$. We show in the following how to construct an assignment $a_{S}$ for $\phi$ from the solution $S$ such that $\operatorname{val}\left(\phi, a_{S}\right)=|S|-8 m-1$. For each variable $x_{i}, S$ cannot contain vertices from both $V^{-}\left(x_{i}\right)$ and $V^{+}\left(x_{i}\right)$ 
since otherwise an edge-vertex has both neighbors inside $S$. Notice also that $S$ cannot contain any vertex $\bar{c}_{j}^{\prime}$, since $\bar{c}_{j}^{\prime}$ is adjacent to the degree one vertex $\bar{c}_{j}$. Similarly $c^{\prime} \notin S$.

If $S$ contains for every $i=1, \ldots, n$ the set $E\left(x_{i}\right)$ and one of the sets $V^{-}\left(x_{i}\right)$ or $V^{+}\left(x_{i}\right)$ then $|S \cap A|=8 \mathrm{~m}$ and we can defined the following assignment $a_{S}$ : $x_{i}=1 \Leftrightarrow\left|S \cap V^{+}\left(x_{i}\right)\right| \neq 0$. In this case, a clause-vertex is in $S$ if and only if the corresponding clause is satisfied by $a_{S}$. Thus, the number of clauses satisfied by $a_{S}$ is exactly $\operatorname{val}\left(\phi, a_{S}\right)=|S|-8 m-1$.

Assume now that $|S \cap A|<8 m$. We show that there exists an other solution $S^{\prime}$ with $\left|S^{\prime}\right| \geq|S|$ such that $\left|S^{\prime} \cap A\right|=8 m$. If a vertex $v \in E\left(x_{i}\right) \backslash S$ for some $i \in\{1, \ldots, n\}$, we can add $v$ in $S$ since $v$ cannot have both neighbors in $S$. Similarly, if $c$ is not in $S$, then we add $c$ in $S$.

Since $|S \cap A|<8 m$, there is at least one vertex either in $V^{+}\left(x_{i}\right) \backslash S$ or in $V^{-}\left(x_{i}\right) \backslash S$ for some $i \in\{1, \ldots, n\}$. Without loss of generality, we only consider vertices in $V^{+}\left(x_{i}\right) \backslash S$. A vertex $v \in V^{+}\left(x_{i}\right) \backslash S$ is either adjacent to $c^{\prime}$ or to a clause-vertex $\bar{c}_{j}^{\prime}$. In the first case we add $v$ in $S$. In the second case, we denote $N\left(\bar{c}_{j}^{\prime}\right)=\left\{v, v^{\prime}, \bar{c}_{j}\right\}$. If $v^{\prime} \in S$ and $\bar{c}_{j} \in S$ then remove $\bar{c}_{j}$ from $S$ and add $v$ instead, otherwise, add $v$ in $S$. Thus, we obtain a new solution $S^{\prime}$ such that $\left|S^{\prime}\right| \geq|S|$ and $\left|S^{\prime} \cap A\right|=8 m$ and in this case as below, we can obtain an assignment $a_{S^{\prime}}$ such that $\left|S^{\prime}\right|-\operatorname{val}\left(\phi, a_{S^{\prime}}\right)=8 m+1$. In particular, if $S^{\prime}$ is an optimal solution, then $\operatorname{opt}(\phi) \geq \operatorname{val}\left(\phi, a_{S^{\prime}}\right)=\operatorname{opt}(I)-8 m-1$ and thus, we have $\operatorname{opt}(I)-\operatorname{opt}(\phi)=8 m+1$ and then $\operatorname{opt}(\phi)-\operatorname{val}\left(\phi, a_{S^{\prime}}\right)=\operatorname{opt}(I)-\left|S^{\prime}\right|$.

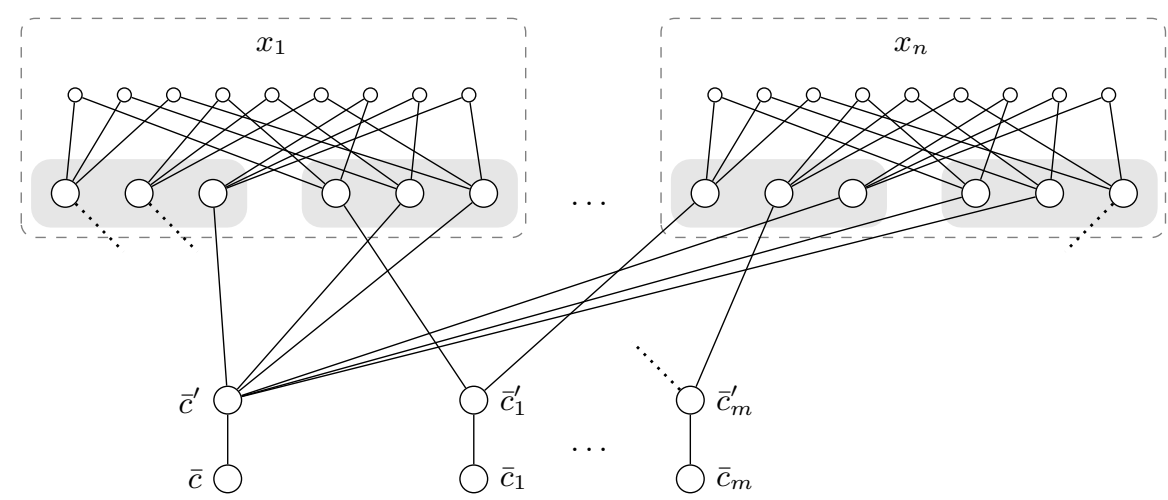

Fig. 1. The construction of $G$.

In the following we propose a PTAS on the class of planar graphs, using the polynomial time algorithm for graphs of bounded treewidth.

Theorem 11. MAX RoBust SET on planar graphs admits a PTAS.

Proof. Given a planar embedding of an input graph, we consider the set of the vertices which are on the exterior face, they will be called level 1 vertices. By 
induction we define level $k$ as the vertices which are on the exterior face when we have removed the vertices of levels smaller than $k$ [2]. A planar embedding is $k$-level if it has no nodes of level greater than $k$. If a planar graph is $k$-level, it has a $k$-outerplanar embedding.

If we want to achieve an approximation within $1+\varepsilon$, let us consider $k=$ $2\left(1+\left\lceil\frac{1}{\varepsilon}\right\rceil\right)$. Let $X_{t}$ be the set of vertices of level $t$ and let $H_{i}, 0 \leq i \leq k-1$, be the graph obtained from $G$ by considering the subgraphs formed by the set of vertices $\bigcup_{t+1 \leq j \leq t+k} X_{j}$, for $t \equiv i(\bmod (k-2))$. The subgraph containing exactly $\bigcup_{t+1 \leq j \leq t+k} X_{j}$ is $k$-outerplanar, and so is $H_{i}$, too.

Since $H_{i}$ is $k$-outerplanar, it has treewidth at most $3 k-1$ [5]. We construct graph $H_{i}^{\prime}$ from $H_{i}$ by attaching a forbidden edge to each vertex on the boundary (that means vertices in $X_{t+1}, X_{t+2}, X_{t+k-1} X_{t+k}$ with $\left.t \equiv i(\bmod (k-2))\right)$. Thus, in each subgraph of $H_{i}^{\prime}$ the vertices in $X_{t+1}, X_{t+2}, X_{t+k-1} X_{t+k}$ cannot take part from any robust set.

On applying Theorem 7, we can efficiently determine an optimal robust set in each subgraph of $H_{i}^{\prime}$. Denote by $S_{i}$ the union of these robust sets. Clearly $S_{i}$ is a robust set on $H_{i}$.

Among $S_{0}, \ldots, S_{k-1}$ we choose the best solution that we denote $S$ and we are going to prove that $S$ is an $(1+\varepsilon)$-approximation of the optimal value on $G$. We can easily show that there is at least one $r, 0 \leq r \leq k-1$ such that at most $\frac{2}{k}$ of vertices in an optimal solution $S_{\text {opt }}$ of $G$ are on levels $X_{t+1}, X_{t+2}, X_{t+k-1} X_{t+k}$ with $t \equiv r(\bmod (k-2)))$. This means that the solution $S_{r}$ obtained by deleting the vertices from levels $X_{t+1}, X_{t+2}, X_{t+k-1} X_{t+k}$ from $S_{o p t}$ will have at least $\left|S_{\text {opt }}\right|\left(1-\frac{2}{k}\right)=\frac{k-2}{k}$ opt vertices. According to our algorithm, $|S| \geq\left|S_{r}\right| \geq \frac{o p t}{1+\varepsilon}$.

The overall running time of the algorithm is $k$ times what we need for graphs of treewidth at most $k$, that is $O\left(k T^{6 k-2} n\right)=n^{O(1 / \varepsilon)}$ where $T=\max _{v \in V} t(v)$.

\section{Conclusion}

In this paper, we introduced the $k$-RoBUST SET problem. We established positive and negative results concerning its parameterized tractability and approximability. However, several questions remain open. For instance, we do not know if the problem is fixed-parameter tractable for parameter treewidth. Another interesting open question is whether $k$-Robust SET WiTH UnANimity is fixedparameter tractable for parameter $k$ when we ask to determine the existence of a robust set of size at least $\left\lceil\frac{n}{3}\right\rceil+k$. Finally, there is room enough for improving the approximability of Max Robust Set With UnANimity.

\section{References}

1. A. Aazami and M. D. Stilp. Approximation algorithms and hardness for domination with propagation. In $A P P R O X$ and RANDOM '07, LNCS 4627, pages 1-15. 2007. 
2. B. S. Baker. Approximation algorithms for NP-complete problems on planar graphs. Journal of the ACM, 41(1):153-180, 1994.

3. O. Ben-Zwi, D. Hermelin, D. Lokshtanov, and I. Newman. Treewidth governs the complexity of Target Set Selection. Discrete Optimization, 8(1):87-96, 2011.

4. P. Berman and M. Karpinski. On some tighter inapproximability results (extended abstract). In 26th International Colloquium on Automata, Languages and Programming (ICALP '99), LNCS 1644, pages 200-209. 1999.

5. H. L. Bodlaender. A partial k-arboretum of graphs with bounded treewidth. Theoretical Computer Science, 209(12):1 - 45, 1998.

6. M. Cesati. The Turing way to parameterized complexity. Journal of Computer and System Sciences, 67(4):654 - 685, 2003.

7. P. Chalermsook and J. Chuzhoy. Resource minimization for fire containment. In Proceedings of the 21th Annual ACM-SIAM Symposium on Discrete Algorithms (SODA '10), pages 1334-1349, 2010.

8. N. Chen. On the approximability of influence in social networks. In Proceedings of the 19th annual ACM-SIAM symposium on Discrete algorithms (SODA '08), pages 1029-1037, 2008.

9. E. J. Cockayne, R. M. Dawes, and S. T. Hedetniemi. Total domination in graphs. Networks, 10(3):211-219, 1980.

10. R. G. Downey and M. R. Fellows. Fixed-parameter tractability and completeness II: On completeness for W[1]. Theoretical Computer Science, 141(12):109 - 131, 1995.

11. R. G. Downey and M. R. Fellows. Parameterized complexity. Springer-Verlag, New York, 1999

12. P. A. Dreyer, Jr. and F. S. Roberts. Irreversible k-threshold processes: Graphtheoretical threshold models of the spread of disease and of opinion. Discrete Applied Mathematics, 157(7):1615-1627, 2009.

13. S. Finbow and G. MacGillivray. The firefighter problem: a survey of results, directions and questions. The Australasian Journal of Combinatorics, 43:57-77, 2009.

14. P. A. Golovach, J. Kratochvil, and O. Suchý. Parameterized complexity of generalized domination problems. Discrete Applied Mathematics, 160(6):780 - 792, 2012.

15. D. Kempe, J. Kleinberg, and É. Tardos. Maximizing the spread of influence through a social network. In Proceedings of the 9th ACM SIGKDD international conference on Knowledge discovery and data mining (KDD '03), pages 137-146, 2003.

16. M. Kimura, K. Saito, and H. Motoda. Blocking links to minimize contamination spread in a social network. ACM Transactions on Knowledge Discovery from Data, $3(2): 9: 1-9: 23,2009$

17. A. Nichterlein, R. Niedermeier, J. Uhlmann, and M. Weller. On tractable cases of target set selection. In 21st International Symposium on Algorithms and Computation (ISAAC '10), LNCS 6506, pages 378-389. 2010.

18. R. Niedermeier. Invitation to Fixed-Parameter Algorithms. Oxford University Press, 2006.

19. C. H. Papadimitriou and M. Yannakakis. Optimization, approximation, and complexity classes. J. Comput. Syst. Sci., 43(3):425-440, 1991. 